\title{
Depleting long noncoding RNA HOTAIR attenuates chronic myelocytic leukemia progression by binding to DNA methyltransferase 1 and inhibiting PTEN gene promoter methylation
}

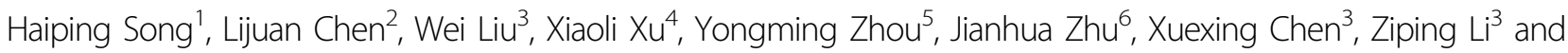
Hao Zhou ${ }^{3}$

\begin{abstract}
Long noncoding RNAs (InCRNAs) are known to play a key role in chronic myelocytic leukemia (CML) development, and we aimed to identify the involvement of the InCRNA HOX antisense intergenic RNA (HOTAIR) in CML via binding to DNA methyltransferase 1 (DNMT1) to accelerate methylation of the phosphatase and tensin homolog (PTEN) gene promoter. Bone marrow samples from CML patients and normal bone marrow samples from healthy controls were collected. HOTAIR, DNMT1, DNMT3A, DNMT3B, and PTEN expression was detected. The biological characteristics of CML cells were detected. The relationship among HOTAIR, DNMT1, and PTEN was verified. Tumor volume and weight in mice injected with CML cells were tested. We found that HOTAIR and DNMT1 expression was increased and PTEN expression was decreased in CML. We also investigated whether downregulated HOTAIR or DNMT1 reduced proliferation, colony formation, invasion, and migration and increased the apoptosis rate of CML cells. Moreover, we tested whether low expression of HOTAIR or DNMT1 reduced the volume and weight of tumors in mice with CML. Collectively, the results of this studied showed that depleted HOTAIR demonstrated reduced binding to DNMT1 to suppress CML progression, which may be related to methylation of the PTEN promoter.
\end{abstract}

\section{Introduction}

Chronic myeloid leukemia (CML) is a myeloproliferative neoplasia triggered by a translocation of reciprocal chromosomes 9 and 22, leading to the generation of a BCR/ABL fusion protein ${ }^{1}$. CML accounts for $20 \%$ of all adult leukemias and is diagnosed at a median age of about 50 years $^{2}$. CML is featured by an initial chronic phase (CP) followed by the development to accelerated (AP) and blast crisis $(\mathrm{BC})$ phase, the latter always resulting in

\footnotetext{
Correspondence: Hao Zhou (zhouhao@hust.edu.cn)

${ }^{1}$ Breast and Thyroid Surgery Center, Union Hospital, Tongji Medical College, Huazhong University of Science and Technology, Wuhan, China

${ }^{2}$ Department of Obstetrics and Gynecology, Union Hospital, Tongji Medical College, Huazhong University of Science and Technology, Wuhan, China

Full list of author information is available at the end of the article

These authors contributed equally: Haiping Song, Lijuan Chen

Edited by M. Agostini
}

patient death ${ }^{3}$. The diagnosis of CML needs the detection of the BCR/ABL oncoprotein, which is present in $95 \%$ of CML patients before the introduction of BCR/ABL as a diagnostic criterion ${ }^{4}$. Tyrosine kinase inhibitors are essential drugs for CML therapy, which could notably ameliorate the prognosis of CML patients ${ }^{5}$. However, most patients only enjoy a short response time, and drug resistance and clinical relapse develop fleetly in the advanced phases of CML post treatment, which are the main limitations for leukemia treatment ${ }^{6}$.

Long noncoding RNAs (lncRNAs) are an important series of non-coding RNAs with a length of 200 nucleotides ${ }^{7}$. LncRNA HOX transcript antisense intergenic RNA (HOTAIR) is transcribed from the homeobox (HOX) C locus, and it suppresses more distal HOXD sites and genes expression on other chromosomes, thus reducing the

\section{(c) The Author(s) 2021}

(c) (i) Open Access This article is licensed under a Creative Commons Attribution 4.0 International License, which permits use, sharing, adaptation, distribution and reproduction cc. in any medium or format, as long as you give appropriate credit to the original author(s) and the source, provide a link to the Creative Commons license, and indicate if changes were made. The images or other third party material in this article are included in the article's Creative Commons license, unless indicated otherwise in a credit line to the material. If material is not included in the article's Creative Commons license and your intended use is not permitted by statutory regulation or exceeds the permitted use, you will need to obtain permission directly from the copyright holder. To view a copy of this license, visit http://creativecommons.org/licenses/by/4.0/. 
expression multiple genes, especially metastasis-inhibitor genes $^{8}$. Epigenetic modulation of HOTAIR has been revealed in advanced $\mathrm{CML}^{9}$. Another study has demonstrated the role of HOTAIR in the acquired resistance to imatinib in CML cells ${ }^{10}$. DNA methylation is the most studied epigenetic modification pattern which is mediated and maintained by DNA methyltransferases (DNMTs), while DNMT1 is necessary for methylation maintenance ${ }^{11}$. It is revealed that DNMT1 and DNMT3A expression are enhanced in cell lines and patients with advanced phase $\mathrm{CML}^{12}$. Another study has demonstrated that DNMT1 mediates abnormal methylation and downregulation of SHP-1 gene in CML cells ${ }^{13}$. As a protein phosphatase and bifunctional lipid, phosphatase and tensin homolog (PTEN) has been reported to attenuate cell growth, phagocytosis, and cytoskeletal remodeling ${ }^{14}$. According to Chen et al., the expression reconstitution of wild-type PTEN dramatically suppresses the invasion, migration, and proliferation abilities of CML K562 cells ${ }^{15}$. Moreover, it is presented that PTEN plays a crucial role in the pathogenesis of $\mathrm{CML}^{16}$. In this study, we aim to identify the involvement of HOTAIR in CML by binding to DNMTs to accelerate the methylation of PTEN promoter.

\section{Results}

HOTAIR and DNMT1 expression are raised, and PTEN expression is reduced in bone marrow of CML patients, and overexpression of HOTAIR is related to poor prognosis of CML patients

RT-qPCR, MSP, and western blot assay displayed that in bone marrow of CML patients, DNMT1, DNMT3A, and DNMT3B were heightened, among which the change of DNMT1 expression was the greatest, so DNMT1 was selected for follow-up experiments. HOTAIR and DNMT1 levels were enhanced, PTEN expression was reduced and PTEN methylation was enhanced in bone marrow of CML patients (all $P<0.05$ ) (Fig. 1A-F).

CML patients were grouped according to WHO classification. RT-qPCR was utilized to detect HOTAIR expression in bone marrow of CML patients, revealing that HOTAIR expression was higher in BC phase than in CP phase (Fig. 1G). However, HOTAIR expression was not changed with age and gender (Fig. 1H, I).

\section{HOTAIR and DNMT1 expression are elevated, and PTEN expression is reduced in CML cells}

In CML cell lines, in contrast with BMCs, HOTAIR and DNMT1 expression were enhanced, and PTEN expression was decreased in CML cells (all $P<0.05$ ), among which $\mathrm{K} 562$ cells showed the greatest difference while KCL-22 cells showed the minimum difference with BMCs (Fig. 2A-D).

In K562 cells, versus the sh-NC group, HOTAIR and DNMT1 expression were decreased, and PTEN expression was raised in the sh-HOTAIR group (all $P<$ 0.05). In comparison to the si-NC group, DNMT1 expression was reduced as well as PTEN expression was enhanced in the si-DNMT1 group (both $P<0.05$ ) (Fig. 2E-H).

In KCL-22 cells, compared to the pc-NC group, HOTAIR and DNMT1 expression were elevated, and PTEN expression was degraded in the pc-HOTAIR group (all $P<0.05)$. Versus the OE-NC group, DNMT1 expression was raised, and PTEN expression was reduced in the OE-DNMT1 group (both $P<0.05$ ) (Fig. 2I-L).

Downregulated HOTAIR or downregulated DNMT1 reduces the proliferation, colony formation and cell cycle progression, as well as increases apoptosis rate of $\mathrm{CML}$ cells

CCK-8, colony formation assays and flow cytometry reported that: in $\mathrm{K} 562$ cells, in contrast to the sh-NC group and the si-NC group, respectively, cell proliferation, the number of colonies, $\mathrm{S}$ and $\mathrm{G} 2 / \mathrm{M}$ phase cells were reduced while G0/G1 phase cells and apoptosis rate were remarkably enhanced in the sh-HOTAIR group and the si-DNMT1 group (all $P<0.05$ ) (Fig. 3A, C, D, G, H, K, L). In KCL-22 cells, versus the pc-NC group and OE-NC group, respectively, cell proliferation, the number of colonies, $\mathrm{S}$ and G2/M phase cells were raised while G0/G1 phase cells and apoptosis rate were reduced in the pc-HOTAIR group and OE-DNMT1 group (all $P<0.05$ ) (Fig. 3B, E, F, I, J, M, N).

\section{Depleted HOTAIR or DNMT1 attenuates invasion and migration of CML cells}

The invasion and migration ability of CML cells were tested by Transwell assay, and the results demonstrated that in K562 cells, the number of invasive and migratory cells was decreased in the sh-HOTAIR group and the siDNMT1 group relative to that in the sh-NC group and the si-NC group (all $P<0.05$ ) (Fig. 4A, B). In KCL-22 cells, by comparison with the pc-NC group and OE-NC group, the number of invasive and migratory cells was enhanced in the pc-HOTAIR group and OE-DNMT1 group (all $P<$ 0.05) (Fig. 4C, D).

\section{HOTAIR binds to DNMT1 to regulate promoter methylation of PTEN}

The results revealed by online analysis site (http:// lncatlas.crg.eu/) and subcellular localization experiment showed that HOTAIR was mainly distributed in the nucleus of K562 and KCL-22 cells (Fig. 5A, B), which was verified by FISH assay (Fig. 5C). RIP assay presented that (Fig. 5D, E) HOTAIR mainly interacted with methyltransferase DNMT1 in K562 cells and KCL-22 cells. ChIP assay revealed that (Fig. 5F, G) DNMT1 was mainly bound to PTEN promoter in K562 cells and KCL-22 cells. In 
A

A

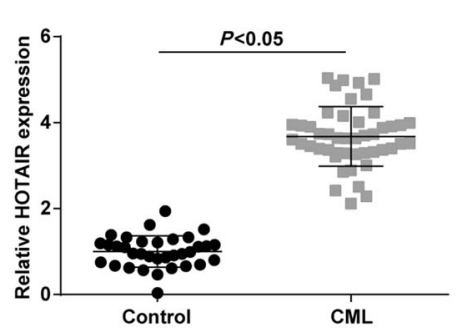

D

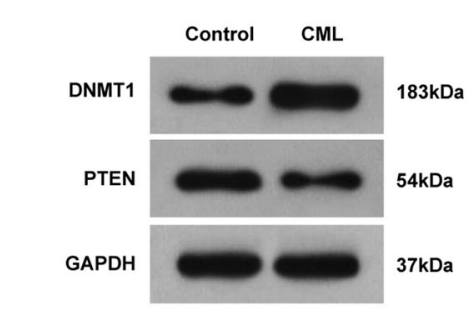

G

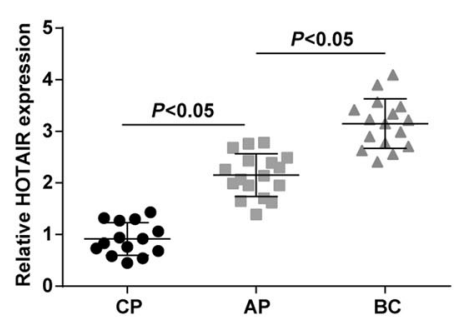

B

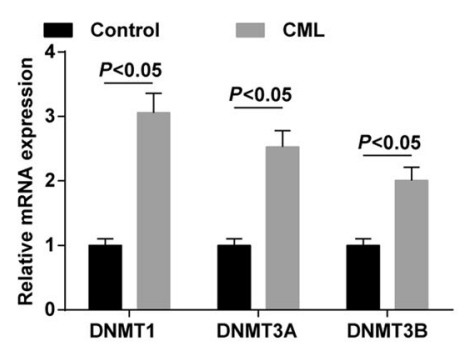

E

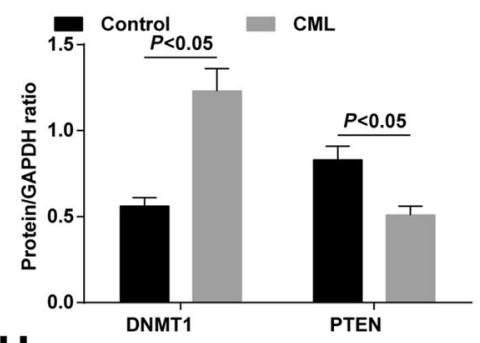

H

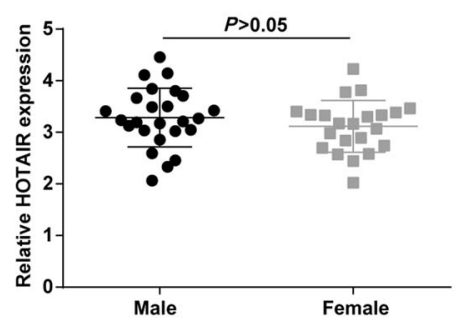

C

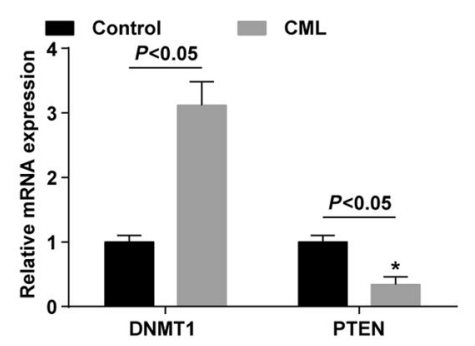

F

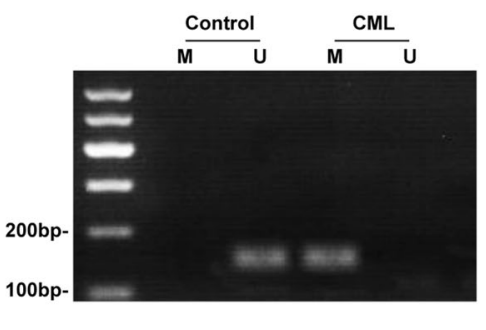

I

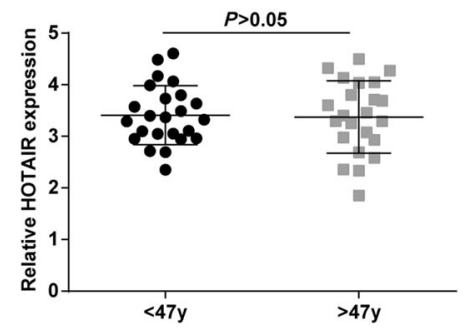

Fig. 1 HOTAIR and DNMT1 expression are raised, and PTEN is reduced in bone marrow of CML patients, and overexpression of HOTAIR is related to poor prognosis of CML patients. A Expression of HOTAIR in patients with CML. B Expression of methyltransferase in patients with CML. C Expression of DNMT1 and PTEN mRNA in patients with CML. D Protein bands of DNMT1 and PTEN in patients with CML. E DNMT1 and PTEN protein expression levels in CML patients. F PTEN methylation in CML patients. G Effect of WHO classification on HOTAIR expression. $\mathbf{H}$ Effect of gender on HOTAIR expression. I Effect of age on HOTAIR expression (control group, $n=34$; CML group, $n=47$; CP group, $n=14 ; \mathrm{Ap}$ group, $n=17$; $\mathrm{BC}$ group, $n=16$; male group, $n=25$; Female group, $n=22$; $<47$ y group, $n=24 ;>47$ y group, $n=23$ ). Measurement data were depicted as mean \pm standard deviation, comparisons between two groups were conducted by independent sample $t$-test.

addition, MSP detection revealed that in K562 cells, versus with the sh-NC and si-NC groups, PTEN methylation level in the sh-HOTAIR and si-DNMT1 groups was reduced (both $P<0.05$ ) (Fig. $5 \mathrm{H}$ ). In $\mathrm{KCL}-22$ cells, with respect to the pc-NC group and OE-NC group, PTEN methylation of pc-HOTAIR group and OE-DNMT1 group was increased (both $P<0.05$ ) (Fig. 5I).

Low expression of HOTAIR or DNMT1 reduces the volume and weight of tumor in mice injected with CML cells

Results of in vivo assay indicated that in K562 cells, versus the sh-NC group and the si-NC group, the tumor volume and weight were reduced in the sh-HOTAIR group and the si-DNMT1 group (all $P<0.05$ ) (Fig. 6A-C). In KCL-22 cells, versus the pc-NC group and OE-NC group, the tumor volume and weight were raised in the
pc-HOTAIR group and OE-DNMT1 group (all $P<0.05$ ) (Fig. 6D-F).

\section{Discussion}

CML is a myeloproliferative disease featured by the $\mathrm{BCR}-\mathrm{ABL}$ fusion gene, which forms a chimeric protein with deregulated tyrosine kinase activity ${ }^{17}$. Also, a recent study has provided a proof that depletion of HOTAIR may play an essential role in ameliorating acquired resistance to imatinib in $\mathrm{CML}^{10}$. It is reported that DNMT1 drives transcriptional downregulation of $\beta$ catenin antagonist Chibby1 linked to the BCR-ABL1 gene of $\mathrm{CML}^{18}$. In a study conducted by Panuzzo et al., it is shown that tumor inhibitor PTEN serves a key role in the pathogenesis of chronic phase CML by non genomic loss of function mechanisms ${ }^{19}$. Our aim is to investigate the 


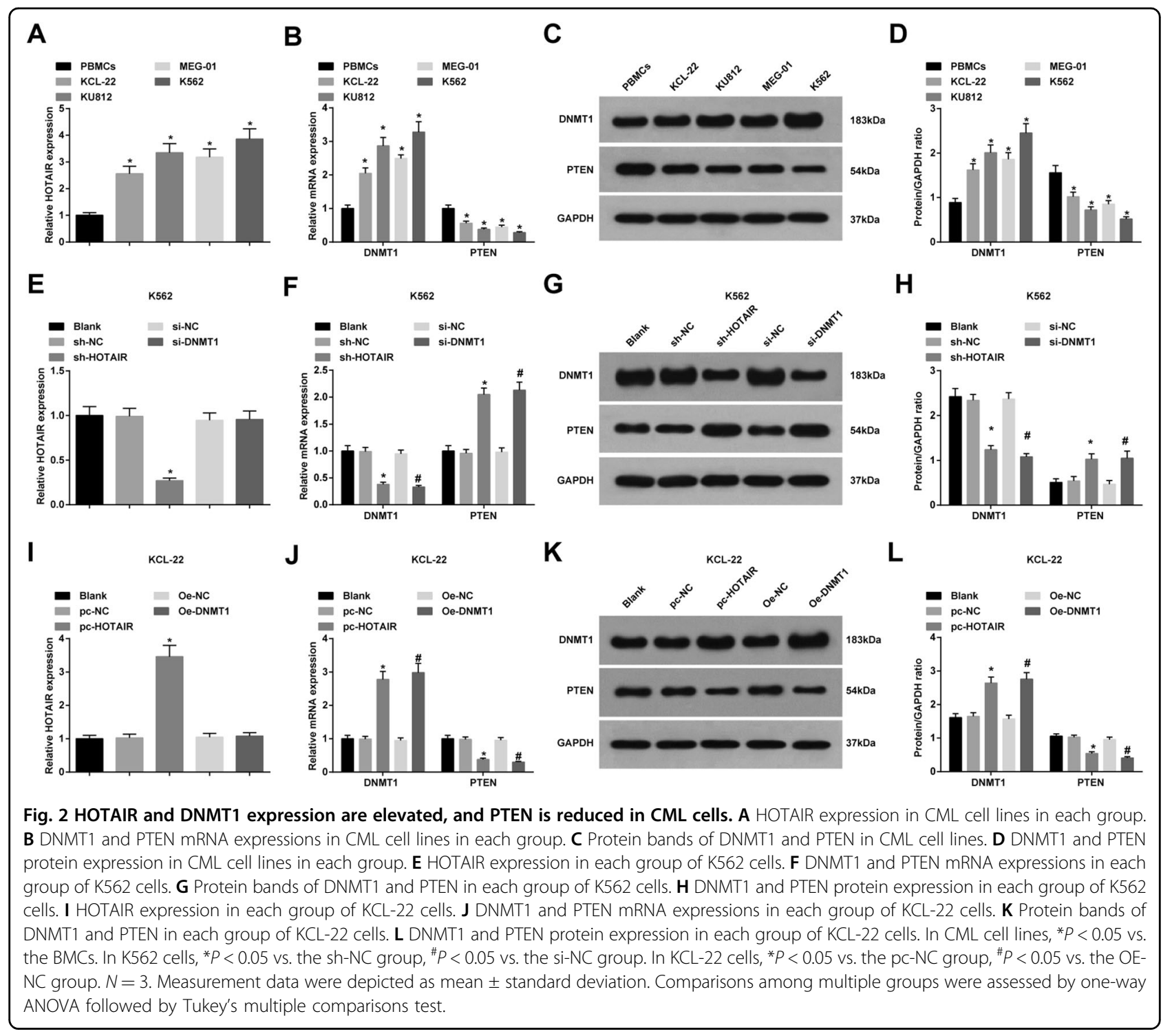

involvement of HOTAIR in CML by binding to DNMTs to promote the methylation of PTEN gene promoter.

In our study, HOTAIR, DNMT1, and PTEN as well as PTEN methylation were detected and the results reported that HOTAIR and DNMT1 expression were raised, while PTEN was reduced in CML cells and bone marrow of CML patients. Meanwhile, upregulated HOTAIR was related to poor prognosis of CML patients. A recent study has presented that HOTAIR expression was markedly elevated in all CML patients relative to that in health donors ${ }^{10}$. Another study has presented that the advanced stage of CML was linked to the high levels of HOTAIR, DNMT1, and DNMT3A ${ }^{9}$. It is presented that DNMT1 expression was dramatically raised in patients in CML-AP and CML-BP versus the controls ${ }^{20}$. It is reported that the expression of PTEN was reduced, whereas DNMT1 was raised in the $\mathrm{AP}$ and $\mathrm{BP}$ of $\mathrm{CML}^{21}$. Furthermore, it is revealed that PTEN protein expression was notably reduced in $\mathrm{CML}$ patients in $\mathrm{AP} / \mathrm{BP}^{22}$. Our study also suggested that HOTAIR bound to DNMT1 in CML cells. A study has reported that HOTAIR and DNMT1 were both up-regulated in $\mathrm{CML}_{\text {cells }}{ }^{9}$, but did not reveal the binding relationship between HOTAIR and DNMT1. Another studies has reported that downregulating HOTAIR degraded DNMT1 protein expression in prostate cancer cells and osteosarcoma cells ${ }^{23,24}$. However, the binding relationship between HOTAIR and DNMT1 in CML needs further study.

Additionally, our data reported that depleted HOTAIR or DNMT1 reduced the proliferation, colony formation, invasion, migration, as well as increased apoptosis rate of CML cells, while low expression of HOTAIR or decreased DNMT1 reduced the volume and weight of tumor in mice injected with CML cells. It has been previously suggested 

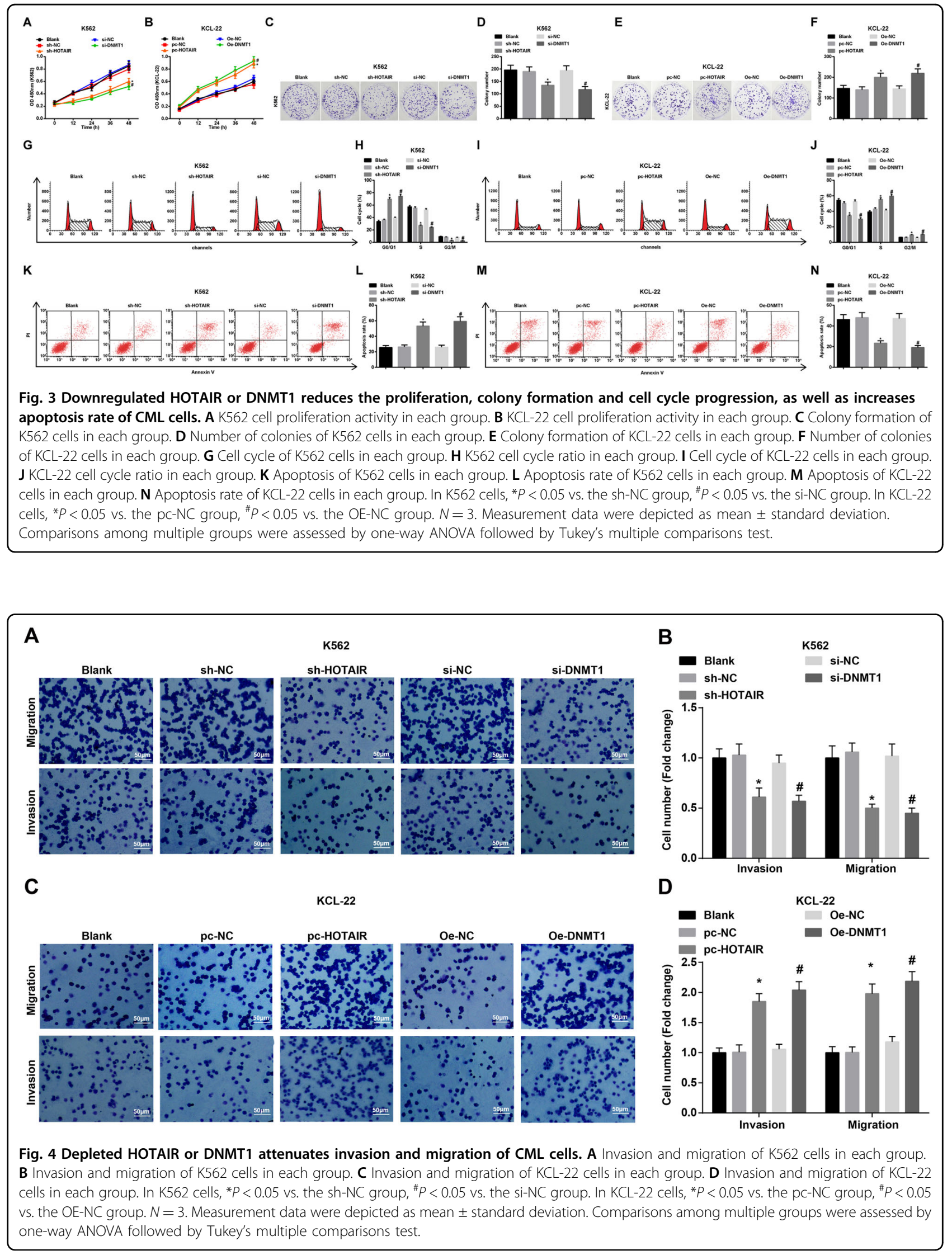


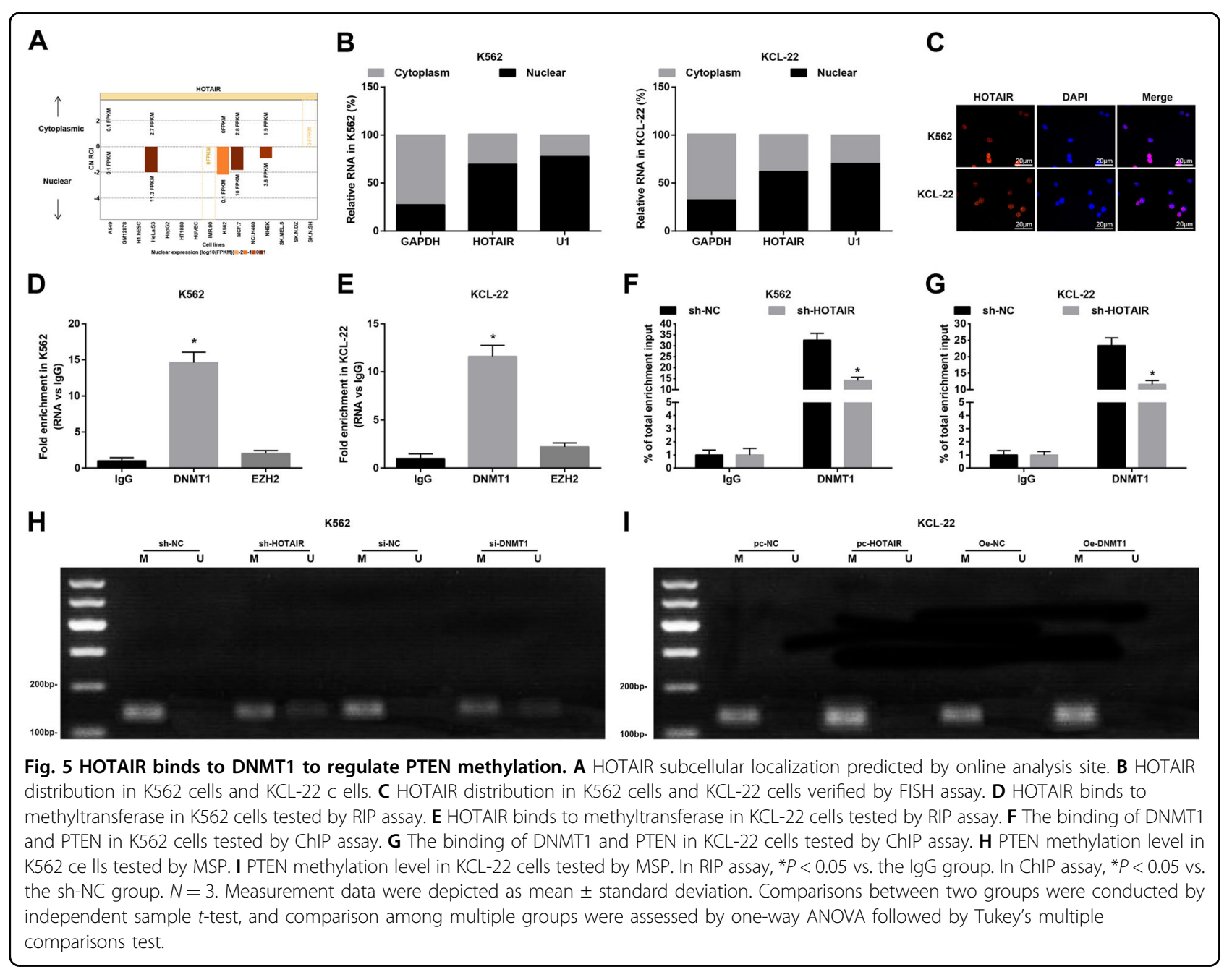

that downregulated HOTAIR attenuated proliferation and enhanced apoptosis in KCL22 and K562 cells ${ }^{9}$. It is reported that depleting HOTAIR in acute myeloid leukemia (AML) reduced cell proliferation and induced apoptosis in vivo and in vitro ${ }^{25}$. It is displayed that low expression of HOTAIR suppressed cell growth and the number of colony formation as well as promoted apoptosis in $\mathrm{AML}^{26}$. Another study also demonstrated that silencing HOTAIR inhibited cell proliferation in leukemia cells ${ }^{27}$. Also, it has been verified that in K562 cells, DNMT1 downregulation led to cell growth inhibition and induction of caspase-3-dependent apoptosis ${ }^{28}$. A prior research has confirmed that inhibition of DNMT1 triggered growth inhibition and apoptosis in multiple myeloma cell lines ${ }^{29}$. Moreover, downregulation of DNMT1 in human tumor xenografts reduced in vivo tumor growth $^{30}$. All these evidence suggests the combined effect of HOTAIR and DNMT1 in CML development.

To briefly conclude, our study provides evidence that depleted HOTAIR could inhibit its binding to DNMT1, thereby suppressing the proliferation and facilitating apoptosis of CML cells, which is related to regulation of methylation of PTEN promoter. These findings provide a new insight into a novel target therapy for CML. Nevertheless, further efforts are still needed.

\section{Materials and methods \\ Study subjects}

From January 2014 to December 2014, bone marrow samples were collected from CML inpatients of Union Hospital, Tongji Medical College, Huazhong University of Science and Technology. All cases were diagnosed by clinical examination, cell morphology analysis, immunology, and histochemical staining. Forty-seven patients had CML, including 25 males and 22 females, with an average age of 47.5 (16.7-78.6) years. The diagnostic type of CML was in accordance with the World Health Organization (WHO) classification criteria ${ }^{31}$. Patients were divided into the CP (14 cases), AP (17 cases), and BC (16 cases) phases. All bone marrow samples were taken from the posterior superior iliac spine. Thirty-four bone marrow samples from the control group were taken from 


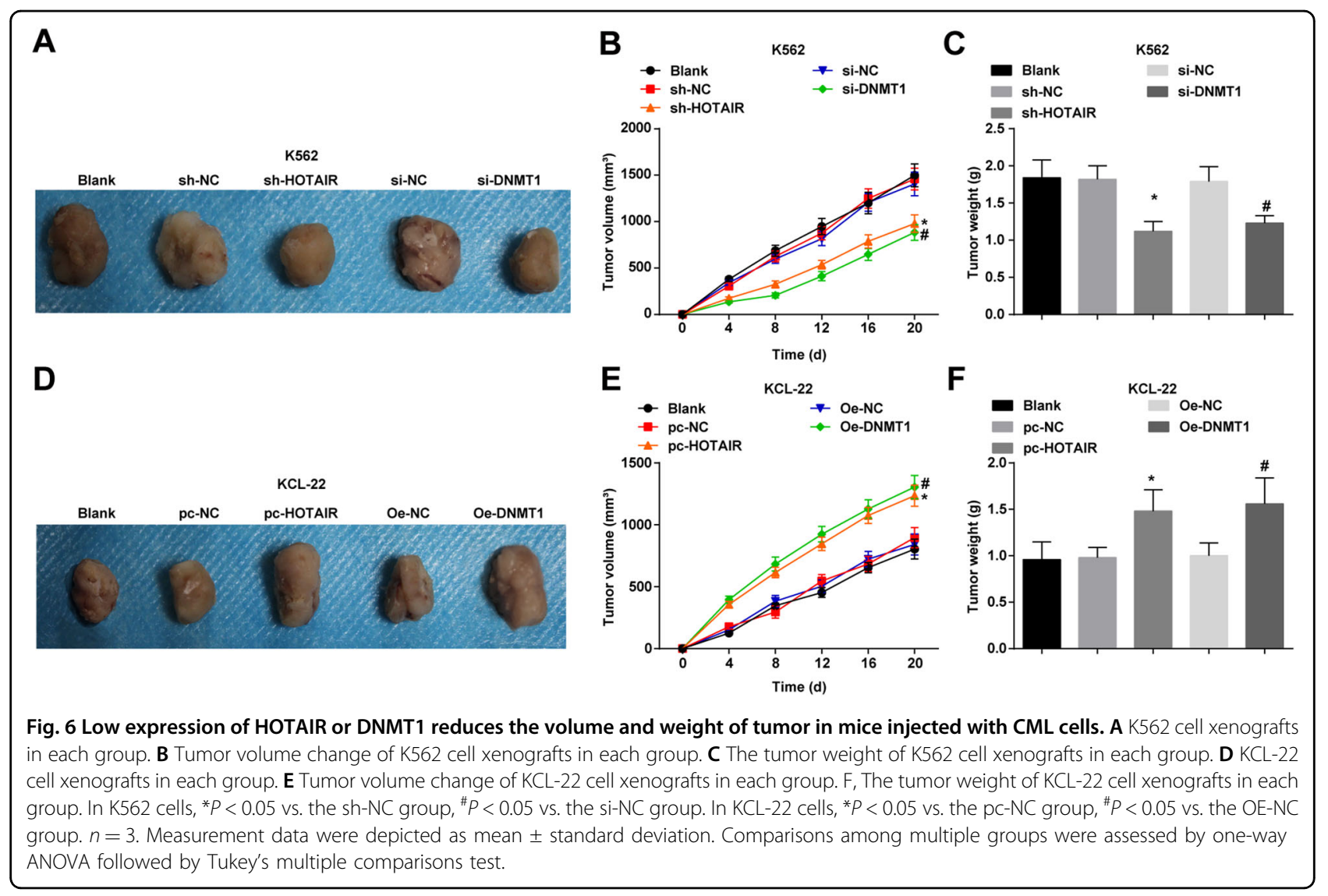

the posterior superior iliac spine of healthy volunteers (18 males and 16 females) with an average age of 38.7 (27.9-72.2) years. Heparin anticoagulant bone marrow (2 $\mathrm{mL} / \mathrm{sample}$ ) was collected, and bone marrow mononuclear cells (BMCs) were amassed and stored at $-80^{\circ} \mathrm{C}$.

\section{Cell culture and screening}

More than $90 \%$ of CML patients have Phl chromosomes in their blood cells, and the $\mathrm{C}$-abl proto-oncogene on the long arm of chromosome 9 is translocated to chromosome 22 to form a BCR/ABL fusion gene ${ }^{32-34}$. KCL-22 cells were established from the pleural effusion of a 32-year-old woman with Philadelphia chromosomepositive CML in blast crisis and contained $t(9 ; 22)$, leading to the BCR-ABL1 e13-a2 (b2-a2) fusion gene and a p53 mutation. K562 cells established from the pleural effusion of a 53-year-old woman with CML in blast crisis carried the BCR-ABL1 e14-a2 (b3-a2) fusion gene; KU812 cells were established from the peripheral blood of a 38-year-old man with CML in myeloid blast crisis and expressed basophilic features and carried $t(9 ; 22)$, leading to the BCR-ABL1 e14-a2 (b3-a2) fusion gene; MEG-01 cells were established from the bone marrow of a 55-year-old man with CML in megakaryocytic blast crisis and carried $t(9 ; 22)$, leading to the BCR-ABL1 e13- a2 (b2-a2) fusion gene. Human CML cell lines (KCL-22, K562, KU812, and MEG-01) containing the BCR/ABL fusion gene were obtained from DSMZ (Braunschweig, Germany). BMCs were used as the control group. All the CML cell lines were cultured in Roswell Park Memorial Institute 1640 medium with $10 \mathrm{~mL} / \mathrm{dL}$ fetal bovine serum (FBS) and $1 \mathrm{~g} / \mathrm{dL}$ penicillin-streptomycin. The medium was changed every 2-3 days. K562 cells with the greatest expression difference compared to BMCs and KCL-22 cells with the minimum expression difference compared to BMCs were selected for subsequent experiments.

\section{Cell grouping and transfection}

K562 and KCL-22 cells were seeded in a 6-well plate at $3 \times 10^{5}$ cells/well. Cells were transfected when they reached approximately $90 \%$ confluence. Transfection was performed with Lipofectamine 2000 reagent according to the manufacturer's instructions (Invitrogen, CA, USA): $3 \mathrm{~mL}$ serum-free medium was utilized to dilute each transfection reaction (all from RiboBio, Guangdong, China, and the final concentration was $50 \mathrm{nM}$ ). Another $3 \mathrm{~mL}$ serum-free medium was utilized to dilute $600 \mu \mathrm{L}$ Lipofectamine 2000. The above two mixtures were mixed and added to cells for $6 \mathrm{~h}$. Then, the medium was changed 
to the corresponding culture medium containing 10\% FBS for subsequent experiments.

K562 cells were assigned into five groups: the blank group (no treatment), short hairpin RNA (sh)-HOTAIR group (transfection of the sh-HOTAIR vector), shnegative control $(\mathrm{NC})$ group (transfection of the shHOTAIR vector NC), small interfering RNA (si)-DNMT1 group (transfection of the si-DNMT1 vector), and si-NC group (transfection of the si-DNMT1 vector NC).

KCL-22 cells were divided into five groups: the blank group (no treatment), pc-HOTAIR group (transfection of the pc-HOTAIR vector), pc-NC group (transfection of the pc-HOTAIR vector NC), overexpression (OE)DNMT1 group (transfection of the OE-DNMT1 vector), and OE-NC group (transfection of the OE-DNMT1 vector $\mathrm{NC}$ ).

\section{Reverse transcription quantitative polymerase chain reaction ( $R T-q P C R$ )}

Total RNA in tissues and cells was extracted by the TRIzol kit (Invitrogen). The purity of RNA was determined by a NanoDrop 2000 spectrophotometer (Thermo Fisher Scientific, MA, USA). Complementary DNA was amplified by a reverse transcription kit (Applied Biosystems, Darmstadt, Germany). The primers (Table 1) were from Sangon (Shanghai, China). Fluorescent quantitative PCR was carried out with the SYBR Premix Ex Taq ${ }^{\mathrm{TM}} \mathrm{II}$ kit (Takara, Shiga, Japan). Fluorescence quantification was performed on a Bio-Rad CFX96 instrument (Bio-Rad, CA, USA). The PCR products were subjected to electrophoresis on a $2 \%$ agarose gel. Glyceraldehyde phosphate

Table 1 Primer sequence.

\begin{tabular}{ll}
\hline Gene & Sequence $\left(\mathbf{5}^{\prime} \rightarrow \mathbf{3}^{\prime}\right)$ \\
\hline HOTAIR & Forward: GGTAGAAAAAGCAACCACGAAGC \\
& Reverse: ACATAAACCTCTGTCTGTGAGTGCC \\
DNMT1 & Forward: AACCTTCACCTAGCCCCAG \\
& Reverse: CTCATCCGATTGGCTCTTCA \\
DNMT3A & Forward: CCTGTGGGGAGCCTCAATGTTA \\
& Reverse: CTTGCAGTTTGGCACATTCC \\
DNMT3B & Forward: CGGCTCTTCTTCGAATTTACC \\
& Reverse: AGAACGGCCGGTCATCAC \\
PTEN & Forward: ATACCAGGACCAGAGGAAACC \\
& Reverse: TTGTCATTATCTGCACGCTC \\
GAPDH & Forward: TGAAGGTCGGAGTCACGG \\
& Reverse: CTGGAAGATGGTGATGGGATT \\
\hline
\end{tabular}

HOTAIR HOX antisense intergenic RNA, DNMT1 DNA methyltransferase 1, DNMT3A DNA methyltransferase 3A, DNMT3B DNA methyltransferase 3B, PTEN phosphatase and tensin homolog, GAPDH glyceraldehyde phosphate dehydrogenase, 18SrRNA $18 \mathrm{~S}$ ribosomal RNA. dehydrogenase (GAPDH) was used as the endogenous reference for HOTAIR, DNMT1, DNMT3A, DNMT3B, and PTEN. Data were analyzed by the $2{ }^{-\Delta \Delta} \mathrm{Ct}$ method. The experiment was repeated three times.

\section{Western blot assay}

Total protein was extracted, and the protein concentration was measured by the bicinchoninic acid method. A sodium dodecyl sulfate separating gel and spacer gel (10\%) were prepared. The sample was mixed with loading buffer and boiled at $100^{\circ} \mathrm{C}$ for $5 \mathrm{~min}$. The protein was separated by electrophoresis and transferred to a nitrocellulose membrane; the membrane was sealed and incubated with primary antibodies against DNMT1 (1:1000, Abcam, MA, USA), PTEN (1:1000), and GAPDH (1:1000, Cell Signaling Technology, MA, USA) overnight. Then, the membrane was incubated with a secondary immunoglobulin G antibody (IgG, 1:2000, Boster, Hubei, China), labeled with horseradish peroxidase and observed postdevelopment. GAPDH was used as the loading control. The gray value of the band image was detected by the Quantity One software system. The ratio of the gray value of the target band to GAPDH was analyzed. The experiment was repeated three times.

\section{Methylation-specific polymerase chain reaction (MSP) assay}

The MSP assay was performed with the DNeasy Tissue Kit according to the manufacturer's instructions (Qiagen company, Hilden, Germany, No69504). Genomic DNA was extracted from cells and stored at $-20^{\circ} \mathrm{C}$. The extracted DNA samples were in the range of 1.7-1.9. The DNA sample was treated with sulfite and purified by the CpGenome (tm) DNA Modification Kit (Chemicon International Inc., CA, USA). Methylated modified PTEN DNA was used as the template for PCR amplification with two pairs of primers (Invitrogen), PTEN methylation (forward: 5'-GGTTTCGGAGGTCGTCGGC-3'; reverse: 5'-CAACCGAATAATAACTACTACGACG-3' [amplification length was $155 \mathrm{bp}$ ]) and non-methylation (forward: 5'-TGGGTTTTGGAGGTTGTTGGT-3'; reverse: $5^{\prime}$-AC TTAACTCTAAACCACAACCA- $3^{\prime}$ [amplification length was $173 \mathrm{bp}])$. Water was used as the NC. The PCR products $(5 \mu \mathrm{L})$ were subjected to electrophoresis on a $3 \%$ agarose gel and observed under an ultraviolet lamp. The experiment was repeated three times.

\section{RNA-binding protein immunoprecipitation (RIP)}

Cells were lysed, and the same amounts of RIP lysate and the corresponding antibodies (DNMT1, 1:200, Abcam), EZH2 (1:100) and IgG (1:1000, both from Cell Signaling Technology) were loaded onto magnetic beads. Then, the bound RNA was purified and analyzed by RTqPCR. The experiment was repeated three times. 


\section{Chromatin immunoprecipitation (ChIP) assay}

Cells transfected with sh-HOTAIR were cultured for 1 day, and then, ChIP assays of K562 and KCL-22 cells were carried out with EZ-Magna ChIP reagent (Millipore, MA, USA). A nonspecific IgG antibody (1:1500, Cell Signaling Technology) was employed as the NC. PTEN gene fragments were quantified by qPCR. Hot-Start Taq polymerase (Takara) was used in a $20 \mu \mathrm{L}$ PCR reaction. The PCR products $(10 \mu \mathrm{L})$ and a 100-bp DNA maker were separated by $2 \%$ agarose electrophoresis for comparison of abundance. The experiment was repeated three times.

\section{Subcellular localization experiment}

The subcellular localization of HOTAIR was predicted by a bioinformatics website (http://lncatlas.crg.eu/). K562/ KCL-22 nuclei and cytoplasms were separated by the PARIS kit (Thermo Fisher Scientific). The relative expression of HOTAIR was verified by RT-qPCR. The experiment was repeated three times.

\section{Fluorescence in situ hybridization (FISH) assay}

Slides $(10 \mathrm{~mm} \times 10 \mathrm{~mm})$ were placed in 24-well plates. Well-grown K562 and KCL-22 cells were seeded at $6 \times$ $10^{3}$ cells/well. FISH assays were performed with LncRNA FISH Probe Mix (Red) and its matching kit (RiboBio). The cells were suspended in a $4{ }^{\circ} \mathrm{C}$ paraformaldehyde solution, cleaned with $0.1 \%$ Triton X-100, prehybridized with prehybridization solution, hybridized with a HOTAIR probe, rinsed with $2 \times$ sodium citrate buffer, stained with the $4^{\prime}$ 6-diamidino-2-phenylindole fluorescent dye, and finally, observed under a fluorescence microscope. The experiment was repeated three times.

\section{Cell counting kit (CCK)-8 assay}

Cell viability was tested by the CCK- 8 kit (Beyotime Institute of Biotechnology, Shanghai, China). Cells $(5 \times$ $10^{3}$ cells, $100 \mu \mathrm{L}$ ) at $80 \%$ confluence were cultured in a 96well plate and then incubated for $3 \mathrm{~h}$ in CCK-8 reagent after culturing for $0,12,24,36$, and $48 \mathrm{~h}$. Optical density (Multiscan FC, Thermo Fisher Scientific) was detected at $450 \mathrm{~nm}$. The experiment was repeated three times.

\section{Colony formation assay}

Cells were trypsinized, and single cells were resuspended. Cells ( 4000 cells/group) were cultured for 14 days in a $60-\mathrm{mm}$ culture dish with $10 \%$ FBS. Colonies were fixed, dyed with $0.5 \%$ crystal violet staining solution, imaged, and counted under a microscope. The experiment was repeated three times.

\section{Transwell assay}

Diluted Matrigel $(50 \mu \mathrm{L})$ was spread on the upper chamber of a Transwell chamber. Cells were suspended in serum-free medium, and a cell suspension $(200 \mu \mathrm{L}, 2 \times$
$10^{5}$ cells $\left./ \mathrm{mL}\right)$ was added to the upper chamber $(600 \mu \mathrm{L}$ cell medium with $10 \%$ FBS was added to the lower chamber). After culturing for $48 \mathrm{~h}$, cells were fixed with absolute methanol and dyed with $0.1 \%$ crystal violet staining solution. Six fields of view were randomly selected under an inverted microscope to count the number of cells that crossed the membrane. The same method was used to detect cell migration, except that the upper chamber was not coated with Matrigel. The experiment was repeated three times.

\section{Flow cytometry}

Cell cycle: Cells were prepared as single-cell suspensions, seeded in a 6-well plate at $1 \times 10^{5}$ cells/well and fastened with $70 \%$ cold ethanol overnight. All steps were performed according to the instructions of the cell cycle detection kit (Dojindo Laboratories, Kumamoto, Japan). The propidium iodide (PI) staining method was adopted with a BD FAC Calibur flow cytometer to detect the cell cycle.

Cell apoptosis: Cells were trypsinized, centrifuged and resuspended. Cells were suspended in $100 \mu \mathrm{L} 1 \times$ binding buffer, mixed with $5 \mu \mathrm{L}$ annexin V-fluorescein isothiocyanate and $5 \mu \mathrm{L}$ PI staining solution for $10 \mathrm{~min}$, mixed with $400 \mu \mathrm{L} 1 \times$ binding buffer and detected by a flow cytometer. The experiment was repeated three times.

\section{Tumor xenograft in nude mice}

A total of 30 female BALB/c-nu/nu nude mice aged 4-5 weeks weighing 16-18g were obtained from the Animal Research Center of the Chinese Academy of Sciences (Shanghai, China). Mice were kept at a constant temperature of $18-22^{\circ} \mathrm{C}$ with a constant humidity of $50-80 \%$ and were provided free access to food and water. Mice were distributed into ten groups $(n=3)$. One day before the experiment, nude mice were exposed to a SliPrecise linear accelerator (Elekta Instruments AB, Stockholm, Sweden). The total dose was $350 \mathrm{cGy}$ per nude mouse to increase the rate of successful cell transplantation. At a cell density of $5 \times 10^{7}$ cells $/ \mathrm{mL}$, the cell suspension $\left(1 \times 10^{7}\right.$ cells $\left./ 200 \mu \mathrm{L}\right)$ was injected subcutaneously into the axillary back of each nude mouse. After 3-5 days, tumor growth was observed. Nude mice were weighed, and tumor volume was measured every 4 days. Nude mice were euthanized 20 days after injection.

\section{Statistical analysis}

All data were explicated by SPSS 21.0 software (IBM Corp. Armonk, NY, USA). The measurement data were conveyed by mean \pm standard deviation. The data distribution was tested for normality. Comparison between two groups was conducted by independent sample $t$-test, and comparison among multiple groups was conducted by one-way analysis of variance (ANOVA) followed by 
Tukey's multiple comparisons test. The association of HOTAIR expression with clinicopathological features of CML was determined by chi-square test. $P$ value $<0.05$ was indicative of statistically significant difference.

\section{Acknowledgements}

We would like to acknowledge the reviewers for their helpful comments on this paper

\section{Author details}

'Breast and Thyroid Surgery Center, Union Hospital, Tongji Medical College, Huazhong University of Science and Technology, Wuhan, China. ${ }^{2}$ Department of Obstetrics and Gynecology, Union Hospital, Tongji Medical College, Huazhong University of Science and Technology, Wuhan, China. Institute of Hematology, Union Hospital, Tongii Medical College, Huazhong University of Science and Technology, Wuhan, China. ${ }^{4}$ Department of Hematology, Foshan No.1 People's Hospital, Fosan, China. ${ }^{5}$ Department of Hematology, The Affiliated Tianyou Hospital, Wuhan University of Science and Technology, Wuhan, China. ${ }^{6}$ Laboratory of Clinical Immunology, Wuhan No. 1 Hospital, Tongji Medical College, Huazhong University of Science and Technology, Wuhan, China

\section{Author contributions}

Hao Zhou contributed to study design; Haiping Song and Lijuan Chen contributed to manuscript editing; Wei Liu, Xiaoli $\mathrm{Xu}$, and Yongming Zhou contributed to experimental studies; Jianhua Zhu, Xuexing Chen, and Ziping Li contributed to data analysis.

\section{Funding}

This work was supported by grants from the National Natural Science Foundation of China (81470348, 81502222).

\section{Ethics statement}

The study was endorsed by the Institutional Review Board of Union Hospital, Tongji Medical College, Huazhong University of Science and Technology and followed the tenets of the Declaration of Helsinki. Participants provided written informed consent to participate in this study. All animal experiments were tally with the Guide for the Care and Use of Laboratory Animal by International Committees.

\section{Conflict of interest}

The authors declare no competing interests.

\section{Publisher's note}

Springer Nature remains neutral with regard to jurisdictional claims in published maps and institutional affiliations.

Received: 4 September 2020 Revised: 1 March 2021 Accepted: 3 March 2021

Published online: 03 May 2021

\section{References}

1. Zhou, H. et al. Combined inhibition of beta-catenin and Bcr-Abl synergistically targets tyrosine kinase inhibitor-resistant blast crisis chronic myeloid leukemia blasts and progenitors in vitro and in vivo. Leukemia 31, 2065-2074 (2017).

2. Azad, N. A. et al. Prognostic implication of BCR-ABL fusion transcript variants in chronic myeloid leukemia (CML) treated with imatinib. a first of its kind study on CML patients of kashmir. Asian Pac. J. Cancer Prev. 19, 1479-1485 (2018).

3. De Barros, S. et al. The impact of chronic myeloid leukemia on employment: the French prospective study. Ann. Hematol. 98, 615-623 (2019).

4. Di Felice, E. et al. The impact of introducing tyrosine kinase inhibitors on chronic myeloid leukemia survival: a population-based study. BMC Cancer $\mathbf{1 8}$ 1069 (2018)
5. Ishida, T. et al. The clinical outcomes of chronic myeloid leukemia patients harboring alternatively spliced BCR-ABL variants. Hematology 24, 49-51 (2019).

6. Cai, H., Qin, X. \& Yang, C. Dehydrocostus lactone suppresses proliferation of human chronic myeloid leukemia cells through Bcr/Abl-JAK/STAT signaling pathways. J. Cell. Biochem. 118, 3381-3390 (2017).

7. Rajagopal, T., Talluri, S., Akshaya, R. L. \& Dunna, N. R. HOTAIR LncRNA: a novel oncogenic propellant in human cancer. Clin. Chim. Acta. 503, 1-18 (2020).

8. Zhang, Y. et al. Upregulation of PUM1 expression in preeclampsia impairs trophoblast invasion by negatively regulating the expression of the IncRNA HOTAIR. Mol. Ther. 28, 631-641 (2020).

9. Li, Z. \& Luo, J. Epigenetic regulation of HOTAIR in advanced chronic myeloid leukemia. Cancer Manag. Res. 10, 5349-5362 (2018).

10. Wang, $\mathrm{H}$. et al. The role of long noncoding RNA HOTAIR in the acquired multidrug resistance to imatinib in chronic myeloid leukemia cells. Hematology 22, 208-216 (2017).

11. Guo, Q. et al. lodine excess did not affect the global DNA methylation status and DNA methyltransferase expression in T and B lymphocytes from NOD.H-2 (h4) and Kunming mice. Int. Immunopharmacol. 55, 151-157 (2018).

12. Zhang, $X$. et al. Research on the epigenetic regulation mechanism of the PTPN6 gene in advanced chronic myeloid leukaemia. Br. J. Haematol. 178 728-738 (2017)

13. Li, Y., Liu, X., Guo, X., Liu, X. \& Luo, J. DNA methyltransferase 1 mediated aberrant methylation and silencing of SHP-1 gene in chronic myelogenous leukemia cells. Leuk. Res. 58, 9-13 (2017).

14. Shi, Y. et al. Downregulation of PTEN promotes podocyte endocytosis of lipids aggravating obesity-related glomerulopathy. Am. J. Physiol. 318, F589-F599 (2020).

15. Zhiyong, C., Wentong, L., Xiaoyang, Y. \& Ling, P. PTEN's regulation of VEGF and VEGFR1 expression and its clinical significance in myeloid leukemia. Med. Oncol. 29, 1084-1092 (2012).

16. Morotti, A. et al. HAUSP compartmentalization in chronic myeloid leukemia. Eur. J. Haematol. 94, 318-321 (2015).

17. Yin, X. et al. Histone demethylase RBP2 mediates the blast crisis of chronic myeloid leukemia through an RBP2/PTEN/BCR-ABL cascade. Cell. Signal. 63, 109360 (2019).

18. Leo, E. et al. DNA methyltransferase 1 drives transcriptional down-modulation of beta catenin antagonist Chibby1 associated with the BCR-ABL1 gene of chronic myeloid leukemia. J. Cell. Biochem. 116, 589-597 (2015).

19. Panuzzo, C. et al. BCR-ABL promotes PTEN downregulation in chronic myeloid leukemia. PLoS ONE 9, e110682 (2014).

20. Li, Z. Y. et al. The long noncoding RNA MEG3 and its target miR-147 regulate JAK/STAT pathway in advanced chronic myeloid leukemia. EBioMedicine 34, 61-75 (2018).

21. Li, Z., Yang, L., Liu, X., Nie, Z. \& Luo, J. Long noncoding RNA MEG3 inhibits proliferation of chronic myeloid leukemia cells by sponging microRNA21. Biomed. Pharmacother. 104, 181-192 (2018).

22. Huang, F. F. et al. PTEN regulates BCRP/ABCG2 and the side population through the PI3K/Akt pathway in chronic myeloid leukemia. PLOS ONE $\mathbf{9}$ e88298 (2014).

23. Xiang, S. et al. HOTAIR-mediated reciprocal regulation of EZH2 and DNMT1 contribute to polyphyllin I-inhibited growth of castration-resistant prostate cancer cells in vitro and in vivo. Biochim. Biophys. Acta 1862, 589-599 (2018).

24. Li, X. et al. A novel interplay between HOTAIR and DNA methylation in osteosarcoma cells indicates a new therapeutic strategy. J. Cancer Res. Clin. Oncol. 143, 2189-2200 (2017).

25. Wang, S. L., Huang, Y., Su, R. \& Yu, Y. Y. Silencing long non-coding RNA HOTAIR exerts anti-oncogenic effect on human acute myeloid leukemia via demethylation of HOXA5 by inhibiting Dnmt3b. Cancer Cell Int. 19, 114 (2019).

26. Xing, C. Y. et al. Long non-coding RNA HOTAIR modulates C-KIT expression through sponging miR-193a in acute myeloid leukemia. FEBS Lett. 589 1981-1987 (2015)

27. Hao, S. \& Shao, Z. HOTAIR is upregulated in acute myeloid leukemia and that indicates a poor prognosis. Int. J. Clin. Int. J. Clin Exp. Pathol. 8, 7223-7228 (2015).

28. Kaufman-Szymczyk, A. Majda, K. Szulawska-Mroczek, A., FabianowskaMajewska, K. \& Lubecka, K. Clofarabinephytochemical combination exposures in CML cells inhibit DNA methylation machinery, upregulate tumor suppressor genes and promote caspasedependent apoptosis. Mol. Med. Rep. 20, 3597-3608 (2019). 
29. Harada, T. et al. HDAC3 regulates DNMT1 expression in multiple myeloma: therapeutic implications. Leukemia 31, 2670-2677 (2017).

30. Thottassery, J. V. et al. Novel DNA methyltransferase-1 (DNMT1) depleting anticancer nucleosides, 4'-thio-2'-deoxycytidine and 5-aza-4'-thio-2'-deoxycytidine. Cancer Chemother. Pharmacol. 74, 291-302 (2014).

31. Vardiman, J. W., Harris, N. L. \& Brunning, R. D. The World Health Organization (WHO) classification of the myeloid neoplasms. Blood $\mathbf{1 0 0}$, 2292-2302 (2002).
32. Huang, X., Chen, Z., Ni, F., Ye, X. \& Qian, W. Shikonin overcomes drug resistance and induces necroptosis by regulating the miR-92a-1-5p/MLKL axis in chronic myeloid leukemia. Aging 12, 17662-17680 (2020).

33. Takawira, C. et al. Association of chronic myelogenous (Basophilic) leukemia and the BCR/ABL mutation in a Yucatan Barrow (Sus scrofa domestica). Front. Vet. Sci. 7, 575199 (2020)

34. Cetin, Z., Ilker Saygili, E. \& Yilmaz, M. Crosstalk between CML cells with HUVECS and BMSCs through CML derived exosomes. Front. Biosci. 26, 444-467 (2021). 